\title{
High Power Sub-Picosecond Pulsed SWIR Source Based on Thulium Assisted Raman Wavelength Shifting
}

\author{
Steevy Cordette ${ }^{1}$, Svyatoslav Kharitonov ${ }^{1}$, Adrien Billat ${ }^{1}$, and Camille-Sophie Brès ${ }^{1}$ \\ 1. Photonic Systems Laboratory, Ecole Polytechnique Fédérale de Lausanne (EPFL) CH-1015 Lausanne, Switzerland \\ steevy.cordette@epfl.ch
}

\begin{abstract}
We demonstrate $1.5 \mathrm{~W}$ sub-picosecond pulsed source near $2 \mu \mathrm{m}$. Cavity-less source is based on integrated association of $3^{\text {rd }}$ order Raman wavelength-shifting and thulium amplification. Coherent spectrum of $61.5 \mathrm{~nm}$ with $49 \%$ pump power conversion efficiency is achieved.

OCIS codes: (230.2285) Fiber devices and optical amplifiers; (060.4370) Nonlinear optics, fibers; (140.3070) Infrared and far-infrared lasers
\end{abstract}

\section{Introduction}

The short-wave infrared (SWIR) spectral region has gathered significant interest during the past years as it is well suited for sensing and communication applications [1]. Sources based on classical fiber laser architectures have recently been demonstrated [2]. Alternative schemes based on parametric conversion in silica fibers, offering the advantages of a cavity-less design, have been reported these past years [3]. Relatively low power continuous wave $(\mathrm{CW})$ generation as well as low repetition rate (of the order of the $\mathrm{MHz}$ ) and high peak power pulsed regimes have been demonstrated $[4,5,6,7]$. Recently, a novel approach based on cascaded Raman wavelength shifting was proposed and demonstrated [8]. In this paper we present a pulsed SWIR fiber source based on thulium assisted cascaded Raman wavelength shifting for the enhanced generation of third Raman Stokes order. The $500 \mathrm{MHz}$ repetition rate source of sub-picosecond pulses features an average power of $1.55 \mathrm{~W}$ for a $49 \%$ pump power conversion efficiency.

\section{Experimental setup}

The generation of the SWIR Raman cascade assisted by Thulium relies on the injection of a high peak power pulse into a highly nonlinear fiber (HNLF) in normal dispersion regime, such that a Raman supercontinuum is generated. The $2 \mu \mathrm{m}$ spectral region of the supercontinuum is then directly amplified by pumping a thulium doped fiber (TDF) with the $\mathrm{C}$ and L-band portion of the supercontinuum itself. The experimental setup is sketched in Fig 1.

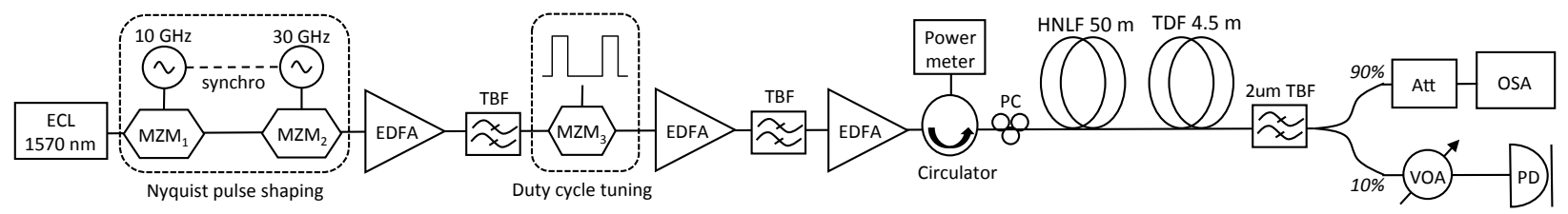

Fig. 1. Experimental setup: ECL: external cavity laser, MZM: Mach-Zehnder modulator, EDFA: erbium doped fiber amplifier, TBF: tunable bandpass filter, PC: polarization controller HNLF: highly nonlinear fiber, TDF: thulium doped fibre, Att: fix attenuator, VOA: variable optical attenuator, OSA: optical spectrum analyzer, PD: photodetector.

The shaping of the pump pulse train is performed in two successive steps. A continuous wave (CW) laser tuned at $1570.25 \mathrm{~nm}$ is first launched into cascaded Mach-Zehnder modulators (MZMs) to obtain a 9.8 ps sinc-shaped Nyquist pulse train with a repetition rate of $10 \mathrm{GHz}$ [9]. The two modulators $\left(\mathrm{MZM}_{1}\right.$ and $\left.\mathrm{MZM}_{2}\right)$ are driven with two synchronized sinusoidal clocks at the respective frequency of $10 \mathrm{GHz}$ and $30 \mathrm{GHz}$. In order to maintain the average power level required for subsequent amplification in a high power erbium doped fiber amplifier (EDFA), this signal is pre-amplified in a low-power amplifier $\left(\mathrm{EDFA}_{1}\right)$ and filtered to remove the amplified spontaneous emission (ASE). Then, the amplified sinc-shaped Nyquist pulse train is modulated by a third intensity modulator $\left(\mathrm{MZM}_{3}\right)$ using a pulse pattern of 1 out of 20 . The resulting $500 \mathrm{MHz}$ return-to-zero pulse train made of 9.8 ps pulses is then amplified in a low-power EDFA and then boosted by a $5 \mathrm{~W}$ high power EDFA. A circulator is positioned right before the HNLF to monitor the back-scattered power due to stimulated Brillouin scattering (SBS) along with a polarization controller necessary to optimize the SWIR generation. Afterward, the pulse train is sent into the HNLF with an average power of $35 \mathrm{dBm}$ and peak power of a $58 \mathrm{dBm}$ sufficient to obtain an auto-stimulated Raman scattering in the HNLF. The $50 \mathrm{~m}$ long segment of HNLF used exhibits a zero dispersion wavelength (ZDW) around $1605 \mathrm{~nm}$, a third-order dispersion of $\beta_{3}=1.5 \cdot 10^{-2} \mathrm{ps}^{3} / \mathrm{km}$, and a nonlinear coefficient of $\gamma=14 \mathrm{~W}^{-1} \cdot \mathrm{km}^{-1}$. By positioning the pump at $1570 \mathrm{~nm}$, the fiber is pumped in the normal dispersion regime and power losses due to 
modulation instability generation are prevented. SBS being circumvented by the use of sub-ns pulses the dominant effect along the propagation is stimulated Raman scattering (SRS) with limited self-phase modulation [10], which leads to the generation of spectral components beyond $2 \mu \mathrm{m}$. Finally, the continuum obtained in the HNLF is injected in a $4.5 \mathrm{~m}$ segment of single mode core-pumped thulium doped fiber (OFS TmDF200). Residual pump as well as a part of the continuum is recycled as pump for the Thulium stage in order to enhance the output power of the proposed SWIR source.

The resulting spectra at the output of the HNLF and the Thulium doped fiber amplifier (TDFA) are monitored on the optical spectrum analyzer (OSA). A $2 \mathrm{~nm}$ wide tunable bandpass filter (TBF) designed to operate around 2 microns (though tunable down to $1800 \mathrm{~nm}$ at the expense of increased losses) is positioned at the output of the TDF to filter part of the spectrum and analyze only a narrow spectral fraction of the generated SWIR source spectrum. This signal is characterized both spectrally on the OSA and temporally on the oscilloscope after detection by a $2 \mu \mathrm{m}$ $9 \mathrm{GHz}$ photodiode. For lack of $2^{\text {nd }}$ order autocorrelator operating in this wavelength range, first order autocorrelation analysis is performed. It must be noted that $27 \mathrm{~dB}$ of attenuation is used for all spectra recording to limit the power sent into the OSA. The attenuation reaches the stated $27 \mathrm{~dB}$ in the telecom-band but dropped to $20 \mathrm{~dB}$ around $1950 \mathrm{~nm}$. This tilt in attenuation is taken into account for all power calculations. Before the photodiode and the autocorrelator, a variable optical attenuator is inserted to avoid photodiode saturation while visualizing the pulses.

\section{Results and discussion}

The spectral comb of the generated $10 \mathrm{GHz}$ repetition rate of the sinc-shaped Nyquist pulses are shown on Fig 2(b), while temporal waveform of the $500 \mathrm{MHz}$ repetition rate pulse train is depicted in Fig 2(a). Note that due to the limited $50 \mathrm{GHz}$ bandwidth of the telecom band photodetector, the impulse response is observed.
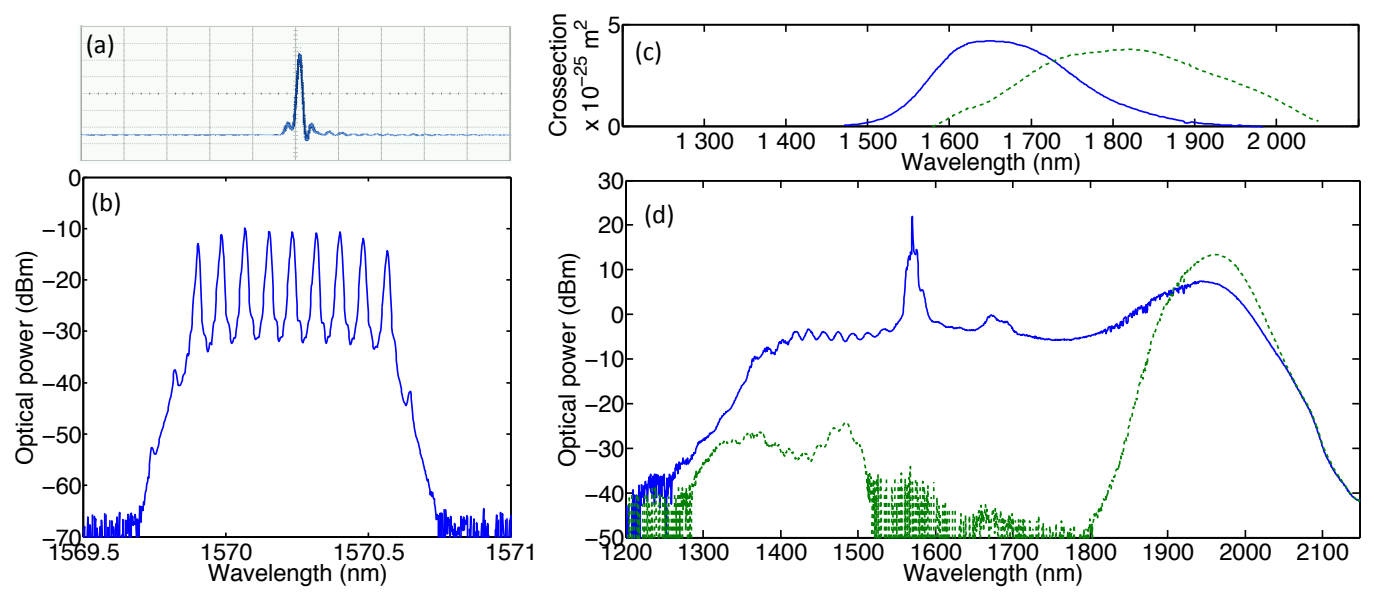

Fig. 2. (a) Temporal waveform of the $500 \mathrm{MHz}$ repetition rate made of $9.8 \mathrm{ps}$ sinc-shaped Nyquist pulses (50GHz photodiode)(100ps/div), (b) Spectrum of the sinc-shaped Nyquist (resolution 0.01nm), (c) Absorption (blue line) and emission (green dashed line) cross-section the TDF, (d) Spectrum at the output of the HNLF (blue line) and the TDFA (green dashed line) for an average pumping power of $35 \mathrm{dBm}$ (resolution $0.01 \mathrm{~nm}$ ). The power is corrected to take into account the attenuation before the OSA.

Figure 2(d) shows spectra recorded at the output of the HNLF and the TDF for an average pump power of $35 \mathrm{dBm}$. The attenuation and its tilt were taken into account and actual powers are displayed. The continuum observed at the output of the HNLF results from cascaded Raman generation up to the third order, as well as soliton compression on the generated Stoke propagating in the anomalous dispersion regime [8]. This second effect leads to the generation of spectral components out of the gain region of the Raman Stokes. At the $3^{\text {rd }}$ Raman spectral region, $10 \mathrm{~dB}$ more power spectral density (PSD) is obtained compared to the $1^{\text {st }}$ and $2^{\text {nd }}$ Raman Stokes spectral region, maximizing the power of the seed signal at the input of the TDFA stage.

The spectrum at the output of the TDFA, obtained for an initial pump pulse train of $500 \mathrm{MHz}$ repetition rate is presented in Fig 2(d) and Fig. 3. Owing to the overlapping of the generated continuum with the wavelength distribution of the absorption and emission cross-section of the TDF (Fig.2 (c))[11], a large portion of the initial Raman pump power (now contained in the $\mathrm{C}$ and $\mathrm{L}$ band spectral range) is recycled as pump for amplification of the $3^{\text {rd }}$ Raman Stoke located within the Thulium emission cross-section. Close to $10 \mathrm{~dB}$ gain is observed on the PSD of the $3^{\text {rd }}$ Raman Stokes order spectral region, reaching a $61.5 \mathrm{~nm}$ bandwidth at the full width half maximum (FWHM). An average power of $1.5 \mathrm{~W}$, corresponding to $49 \%$ of the pump power injected in the HNLF, is measured for a bandwidth at $-10 \mathrm{~dB}$. 

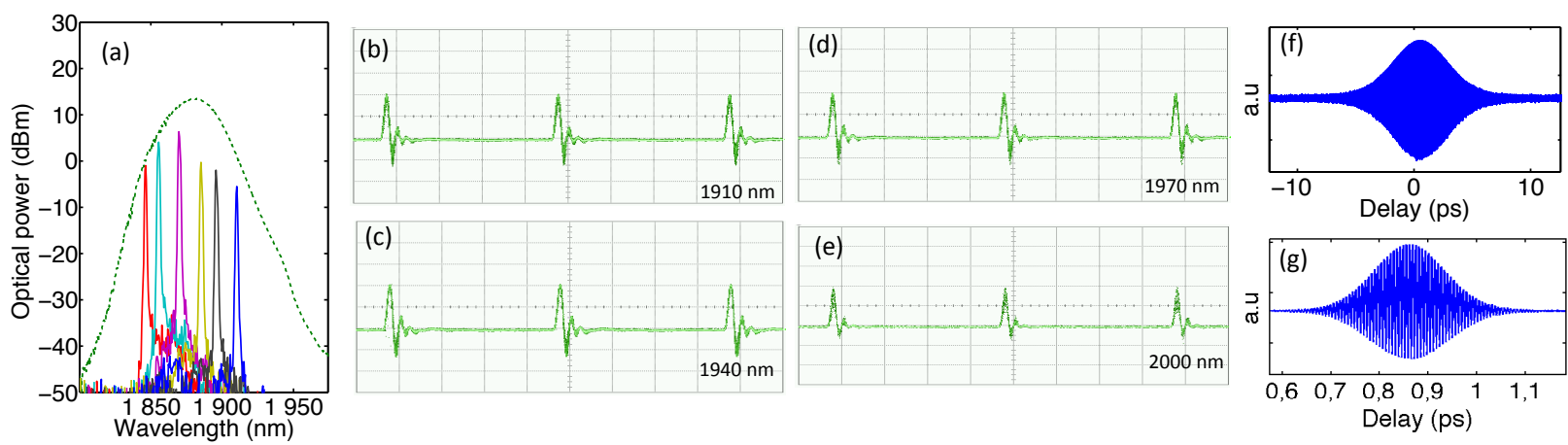

Fig. 3. Superimposed spectra of filtered SWIR source (plain line) and the entire $61.5 \mathrm{~nm}$ SWIR source bandwidth (green dashed line) (a). Temporal waveforms of filtered SWIR source at the wavelength (b) $1910 \mathrm{~nm}$, (c) $1940 \mathrm{~nm}$ and (d) $1970 \mathrm{~nm}$ and (e) $2000 \mathrm{~nm}$ (9 GHz photodiode) $(500 \mathrm{ps} / \mathrm{div})$. (f) First order autocorrelation of the filtered SWIR source at $1910 \mathrm{~nm}$ and for the entire $61.5 \mathrm{~nm}$ SWIR source bandwidth (g).

The SWIR source is temporally characterized by tuning the TBF between $1880 \mathrm{~nm}$ and $2020 \mathrm{~nm}$ and observing the detected SWIR pulse train on the oscilloscope. The superimposed spectra of the filtered SWIR source are shown in Fig. 3(a), where the spectra reflect the wavelength dependent losses of the TBF. Waveforms of four of the detected wavelengths, namely $1910 \mathrm{~nm}, 1940 \mathrm{~nm}, 1970 \mathrm{~nm}$ and $2000 \mathrm{~nm}$ are displayed in Figures 3 (b), (c), (d), and (e), respectively. The resolution of the pulse width is limited by the impulse response of the photodiode, which clearly appears for all measurements. Moreover we observed in the temporal waveform of the filtered SWIR source a slight temporal shift of the pulse with respect to the set wavelength of TBF. This behavior tends to indicate a significant reduction of the chirp, compared to the results reported in [8], such that simple chirp compensation can be envisioned. Figures 2(f) and (g) display the first order autocorrelation measured for the filtered SWIR source at $1910 \mathrm{~nm}$ and for the entire $61.5 \mathrm{~nm}$ bandwidth SWIR source, where a $5 \mathrm{ps}$ and $147 \mathrm{fs}$ FWHM of autocorrelation are respectively obtained. Despite the fact that pulse duration measurement via first order autocorrelation are not exact in the case of chirped pulse, it allows to estimate the pulse width potentially achievable with the proposed SWIR source.

\section{Conclusion}

An integrated combination of a cascaded Raman wavelength shifting and Thulium-doped fiber amplification that outputs $500 \mathrm{MHz}$ repetition rate pulses with high average power in the SWIR band has been demonstrated. A TDF is used to enhance the output power of the $3^{\text {rd }}$ Raman Stokes while also acting as a low pass filter. The un-wanted high frequency component power of the continuum, including the residual Raman pump power, is efficiently recycled as a TDFA pump to achieve a $1.5 \mathrm{~W}$ average power at the output of the presented $61.5 \mathrm{~nm}$-bandwidth SWIR pulsed source for $49 \%$ pump conversion efficiency. This source could potentially provided sub-picosecond down to femto-second pulses thanks to its wide bandwidth when adequate chirp compensation is applied. Furthermore as the $2-\mu \mathrm{m}$ signal is initiated from a Raman process driven in the telecommunication band, such configuration can also be used for higher repetition rates and for the transfer data in SWIR.

This work is supported by the European Research Council under grant agreement ERC-2012- StG 306630-MATISSE.

\section{References}

[1] M. N. Petrovitch et al., "Demonstration of amplified data transmission at $2 \mu \mathrm{m}$ in a low-loss wide bandwidth hollow core photonic bandgap fiber", Opt. Express 21, p. 28559 (2013).

[2] E. M. Dianov et al., "Raman fibre lasers emitting at a wavelength above $2 \mu \mathrm{m}$ ", J. Lightwave Technol. 24, p. 695 (2004).

[3] F. Gholami et al., "All-Fiber Tunable SWIR Parametric Source at $2.4 \mu \mathrm{m} "$, proc. Frontiers in Optics, paper FWW3, New York (2010).

[4] L.-M. Yang et al., "2 $\mu \mathrm{m}$ femtosecond fiber laser at low repetition rate and high pulse energy", Opt. Express 20, p. 5683 (2012).

[5] J. M. Chavez Boggio et al., "Tunable Parametric All-Fiber Short-Wavelength IR Transmitter", J. Lightwave Technol. 28, p. 443 (2010).

[6] H. Hoogland et al., "All-PM coherent $205 \mu \mathrm{m}$ Thulium/Holmium fiber frequency comb source at $100 \mathrm{MHz}$ with up to $5 \mathrm{~W}$ average power and pulse duration down to 135 fs", Opt. Express 21, p. 31390 (2013).

[7] P. Wan et al., "High-energy femtosecond 2- $\mu \mathrm{m}$ fiber laser", Opt. Eng. 53, p. 51508 (2014).

[8] S. Cordette et al., "Widely Tunable Picosecond-Pulsed Source near $2 \mu \mathrm{m}$ based on Cascaded Raman Wavelength Shifting", proc. ECOC, paper P.1.17, Cannes (2014).

[9] M. A. Soto et al., "Optical sinc-shaped Nyquist pulses of exceptional quality", Nat. Communications, 4, 31347 (2013).

[10] M. Duhant et al., "Fourth-order cascaded Raman shift in AsSe chalcogenide suspended-core fiber pumped at $2 \mu \mathrm{m} "$ Opt. Lett. 36, p. 2859 (2011).

[11] S. Agger and J. Povlsen, "Emission and absorption cross section of thulium doped silica fibers," Opt. Express 14, 50-57 (2006). 